\title{
Impacto da Corionicidade nas Complicações Perinatais da Gestação Gemelar
}

\author{
Perinatal Outcome in Relation to Chorionicity in Twin \\ Pregnancy
}

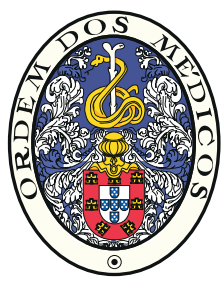

\author{
Marta MACHADO $\otimes^{1}$, Elsa LIMA TEIXEIRA², Lígia Maria FERREIRA², Filipa RODRIGUES ${ }^{1}$, Raquel HENRIQUES ${ }^{3}$, \\ Eulália $\mathrm{AFONSO}^{3}$ \\ Acta Med Port 2017 Jan;30(1):12-16 - http://dx.doi.org/10.20344/amp.7133
}

\section{RESUMO}

Introdução: A incidência da gestação múltipla tem vindo a aumentar em todo o mundo e vários estudos têm demonstrado taxas de morbilidade e mortalidade mais elevadas nos gémeos monocoriónicos comparativamente com os bicoriónicos. Os objetivos deste trabalho foram: caracterizar a população de gémeos fruto de gravidez bifetal nascidos num hospital nível três e avaliar o impacto da corionicidade na morbimortalidade perinatal.

Material e Métodos: Estudo retrospetivo de todos os gémeos fruto de gravidez bifetal nascidos num hospital nível três entre janeiro de 2004 e dezembro de 2013.

Resultados: Neste período nasceram 1051 gémeos, fruto de 540 gestações (26,7\% monocoriónicos; $73,3 \%$ bicoriónicos). Não houve diferença estatisticamente significativa entre os dois grupos no respeitante às complicações obstétricas. No grupo monocoriónico verificou se uma incidência mais elevada de restrição do crescimento intra-uterino $(20,5$ vs $11,3 \%, p<0,001)$, idade materna mais baixa $(29,9$ vs 31,9 anos, $p<0,001)$, idade gestacional mais baixa $(33,4$ vs 34,3 semanas, $p<0,05)$ e peso de nascimento mais baixo (1943 vs $2147 \mathrm{~g}, p<0,001)$. Os gémeos monocoriónicos tiveram uma incidência mais elevada de doença de membrana hialina $(7 \mathrm{vs} 4 \%$, $p<0,05)$, sépsis $(10,3$ vs $5,8 \%, p<0,05)$ e anemia $(9,5$ vs $5,4 \%, p<0,05)$. Não se encontrou diferença estatisticamente significativa relativamente à ocorrência de enterocolite necrotizante, hemorragia intraperiventricular ou retinopatia da prematuridade. A mortalidade perinatal foi mais elevada no grupo monocoriónico $(5,2$ vs $2,9 \%, p<0,05)$.

Discussão: Os gémeos monocoriónicos representam um desafio para obstetras e neonatologistas, devendo a vigilância pré-natal e o parto ser realizados em centros de referência.

Conclusão: A gemelaridade tem atualmente um importante impacto nos nascimentos, pelo que seria interessante desenvolver protocolos que uniformizassem a prática clínica na abordagem a estes recém-nascidos.

Palavras-chave: Complicações na Gravidez; Córion; Gravidez de Gémeos; Resultado da Gravidez

\section{ABSTRACT}

Introduction: The incidence of multiple gestations is increasing worldwide and many studies have shown higher perinatal morbidity and mortality rates in monochorionic twins compared to dichorionic. The aim of this study was to assess the twin population born at a tertiary center and to evaluate the impact of chorionicity on perinatal outcomes of twin pregnancies.

Material and Methods: Retrospective study of all twins born in a tertiary center from January 2004 to December 2013.

Results: In this period, 1051 twins were born, related to 540 gestations ( $26.7 \%$ monochorionic; $73.3 \%$ dichorionic). There was no statistical significant difference between the groups concerning obstetric complications. The monochorionic group had a higher incidence of intrauterine growth restriction $(20.5 \mathrm{vs} 11.3 \%, p<0.001)$, lower mean maternal age (29.9 vs 31.9 years, $p<0.001)$, lower mean gestational age (33.4 vs 34.3 weeks, $p<0.05$ ) and lower mean birth weight (1943 vs $2147 \mathrm{~g}, p<0.001)$. Monochorionic twins had a higher incidence of hyaline membrane disease ( 7 vs $4 \%, p<0.05)$, sepsis $(10.3$ vs $5.8 \%, p<0.05)$ and anemia $(9.5$ vs $5.4 \%$, $p<0.05)$. There were no statistical significant differences concerning necrotizing enterocolitis, intraperiventricular hemorrhage or retinopathy of prematurity. Perinatal mortality was higher in the monochorionic group (5.2 vs $2.9 \%, p<0.05)$.

Discussion: Monochorionic twins represent considerable challenges to both obstetricians and neonatologists and should be monitored and delivered at tertiary centers.

Conclusion: Currently gemelarity has a major impact on total births. It would be interesting to develop protocols to standardize clinical approach to twins

Keywords: Chorion; Pregnancy Complications; Pregnancy Outcome; Pregnancy, Twin

\section{INTRODUÇÃO}

A incidência da gestação múltipla tem vindo a aumentar em todo o mundo, representando atualmente cerca de $3 \%$ - $4 \%$ das gestações,,$^{1-3}$ sobretudo devido ao adiar da idade de maternidade (fator associado a uma maior probabilidade de gravidez múltipla) e ao recurso a técnicas de procriação medicamente assistida (PMA).4-11

A gravidez bifetal representa a maioria dos casos de gravidez múltipla (98\%). ${ }^{1}$ Enquanto a gestação monozigótica monocoriónica (MC) tem uma incidência relativamente constante (3-5:1000), , ${ }^{1,511-13}$ a dizigótica (forçosamente bicoriónica - BC), mais frequente, é influenciada por múltiplos fatores, incluindo hereditariedade, raça/etnia e idade materna. ${ }^{1,4,5,12}$ As técnicas de PMA, conhecidas por aumentar a incidência de gestação dizigótica, parecem também

1. Serviço de Pediatria. Centro Hospitalar do Baixo Vouga. Aveiro. Portugal.

2. Serviço de Pediatria. Centro Hospitalar Tondela-Viseu. Viseu. Portugal.

3. Serviço de Neonatologia. Maternidade Daniel de Matos. Centro Hospitalar e Universitário de Coimbra. Coimbra. Portugal.

$\triangle$ Autor correspondente: Marta Machado. marta.scamachado@gmail.com

Recebido: 27 de outubro de 2015 - Aceite: 21 de março de 2016 | Copyright @ O Ordem dos Médicos 2017 
influenciar a monozigotia. ${ }^{14,15}$ Um estudo recente indica que o risco de monozigotia é superior (na ordem dos 60\%) nas situações de PMA comparativamente com a gestação espontânea. ${ }^{15}$

A maior morbimortalidade materna e fetal/neonatal na gravidez múltipla relativamente à unifetal está relacionada não apenas com o número de fetos mas também com o tipo de corionicidade. ${ }^{1,2,6-8,13}$ Vários estudos têm demonstrado taxas de morbilidade e mortalidade mais elevadas nos gémeos monocoriónicos comparativamente com os bicoriónicos..$^{1,4,9,16}$ Isto pode ser explicado pela ocorrência frequente de anastomoses vasculares entre os fetos e de inserção anómala do cordão umbilical e pela partilha muitas vezes desigual da placenta, fatores responsáveis pela síndrome de transfusão feto-fetal (STFF), restrição de crescimento intra-uterino (RCIU) e crescimento fetal discordante (CFD), com potenciais consequências nefastas para ambos os fetos. ${ }^{4,11-13,16,17}$

Os objetivos deste trabalho foram: caracterizar a população de gémeos fruto de gravidez bifetal nascidos num hospital nível três e avaliar o impacto da corionicidade na morbimortalidade perinatal.

\section{MATERIAL E MÉTODOS}

Foi realizado um estudo descritivo e analítico com análise retrospetiva através da colheita de dados dos registos clínicos de todos os gémeos fruto de gravidez bifetal nascidos num hospital nível três num período de dez anos: entre janeiro de 2004 e dezembro de 2013.
As gestações foram classificadas como MC biamnióticas ou BC de acordo com critérios ecográficos e avaliação histológica da placenta após o parto. Foram excluídas as gestações monoamnióticas.

Para caracterização da evolução clínica, foram definidos/as: RCIU estabelecido por diagnóstico pré-natal de acordo com os critérios utilizados pelo Serviço de Obstetrícia; CFD quando a diferença no peso fetal estimado entre os gémeos era igual ou superior a $20 \%{ }^{1,7,9}$; STFF através de critérios ecográficos ${ }^{10,18}$; parto pré-termo (PPT) quando ocorrido antes das 37 semanas de gestação; hemorragia peri-intraventricular (HPIV) segundo a classificação de Volpe ${ }^{19}$; anemia de acordo com o consenso da Secção de Neonatologia da Sociedade Portuguesa de Pediatria ${ }^{20}$; retinopatia da prematuridade (ROP) de acordo com a classificação internacional de $\mathrm{ROP}^{21}$; mortalidade perinatal englobando os óbitos fetais de 28 ou mais semanas de gestação e os óbitos de nados-vivos com menos de sete dias de idade (como definido pelo Instituto Nacional de Estatística), excluindo anomalias fetais major.

Foram estudadas as seguintes variáveis: idade materna, tipo de conceção (espontânea ou por PMA), corionicidade ( $\mathrm{MC}$ ou $\mathrm{BC}$ ), eventos pré-natais, idade gestacional $(I G)$, peso de nascimento (PN), género, tipo de parto, admissão em Unidade de Cuidados Intensivos Neonatais (UCIN), morbilidade e mortalidade perinatais e neonatais.

Os dados foram avaliados utilizando os testes $\chi^{2}$ e $t$ de Student, para um intervalo de confiança de $95 \%$, através do programa SPSS v. 18.0 .

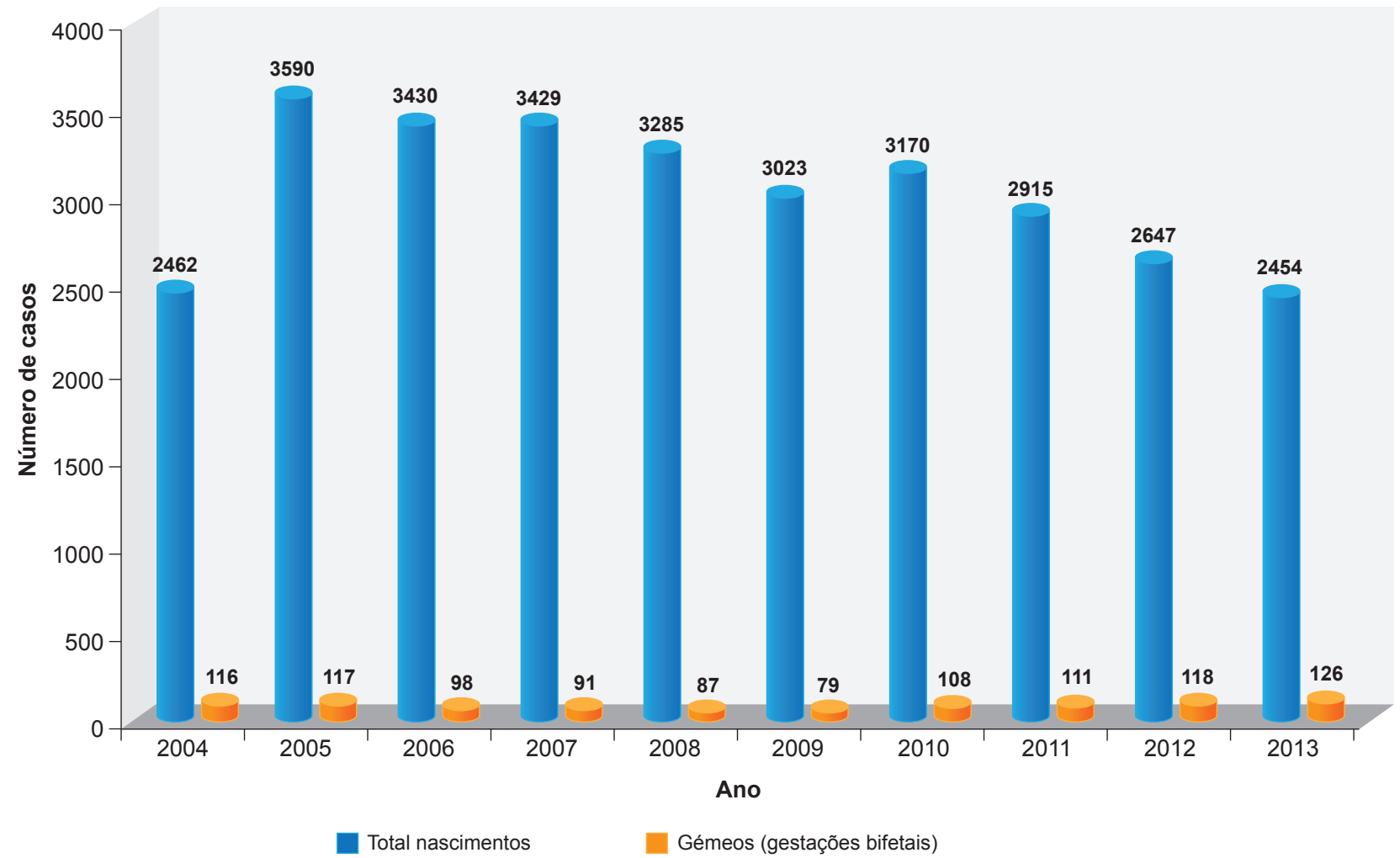

Figura 1 - Representação gráfica do total de RN gémeos fruto de gestações bifetais em relação ao total de nascimentos no nosso centro em cada ano de estudo 


\section{RESULTADOS}

Neste período nasceram 1051 recém-nascidos (RN) gémeos ( $51 \%$ do género feminino), fruto de 540 gestações bifetais (73,3\% BC; $26,7 \%$ MC). Representam 3,5\% dos nados vivos nascidos no nosso centro nesta década (1051 em 30 405), com uma incidência mais baixa de 2007 a 2009 (2,6\%) e mais elevada em 2013 (5,1\%) (Fig. 1).

Na gestação MC a idade materna média foi mais baixa $(29,9$ vs 31,9 anos, $p<0,001)$ e a taxa de gestação espontânea superior $(92,4$ vs $64,9 \%, p<0,001)$. O recurso a técnicas de PMA resultou, na sua maioria (92,7\%), em gestação BC. Mesmo excluindo as gestações fruto de PMA, a idade materna média continua a ser mais baixa na gestação $M C(29,5$ vs 31,3 anos, $p<0,001)$.

Verificou-se uma incidência mais elevada de RCIU na gestação $M C$ (20,5 vs 11,3\%, $p<0,001)$, sem que, no entanto, se tivessem encontrado diferenças estatisticamente significativas entre os dois grupos no respeitante às restantes complicações obstétricas estudadas, nomeadamente diabetes gestacional, patologia do líquido amniótiTabela 1 - Características e complicações materno-obstétricas co (oligoâmnios/hidrâmnios) e ameaça de PPT (Tabela 1). Registaram-se 16 casos de STFF em 144 gestações MC $(11,1 \%)$.

Relativamente ao parto, a incidência de cesariana foi superior na gestação $M C(65,3$ vs $54,2 \%, p<0,05)$ (Tabela 2). A percentagem de PPT na amostra foi elevada $(79,7 \%)$ (Tabela 3), na sua maioria RN pré-termo tardios $(63,2 \%$ com IG entre 34 e 36 semanas), representando $29,7 \%$ do total de RN pré-termo nascidos no nosso centro no mesmo período. Encontrou-se uma diferença significativa na IG média, tendo esta sido inferior no grupo MC $(33,4$ vs 34,3 semanas, $p<0,05)$. Também a média de $\mathrm{PN}$ foi mais baixa neste grupo (1943 vs 2147 gramas, $p<0,001$ ). O peso mínimo foi de 450 gramas e o máximo de 3850 gramas. No global, 16,2\% tinham peso inferior a 1500 gramas (Tabela 3 ), o que corresponde a $27,2 \%$ do total de RN muito baixo peso para esse período. Não se registou diferença significativa no índice de Apgar ao quinto minuto.

Foram admitidos na UCIN 513 gémeos (49\%), correspondendo a $17 \%$ do total de RN admitidos nesse período.

\begin{tabular}{lccc}
\hline & MC & BC & $p$ \\
& $(\mathrm{n}=144)$ & $(\mathrm{n}=396)$ & $\mathbf{0 , 0 0 1}$ \\
\hline Idade materna média (anos) & $\mathbf{2 9 , 9}$ & $\mathbf{3 1 , 9}$ & $\mathbf{0}, \mathbf{0 0 1}$ \\
RCIU (\%) & $\mathbf{2 0 , 5}$ & $\mathbf{1 1 , 3}$ & $>0,05$ \\
Diabetes gestacional (\%) & 2,1 & 6,1 & $>0,05$ \\
Patologia do líquido amniótico (\%) & 5,6 & 4,8 & $>0,05$ \\
Ameaça de PPT (\%) & 2,8 & 6,1 & $>0,05$ \\
Crescimento fetal discordante (\%) & $27,8 \%$ & $20,2 \%$ & \\
\hline
\end{tabular}

Tabela 2 - Principais características dos recém-nascidos

\begin{tabular}{|c|c|c|c|}
\hline & $\begin{array}{c}M C \\
(n=273)\end{array}$ & $\begin{array}{c}\text { BC } \\
(n=778)\end{array}$ & $p$ \\
\hline Parto por cesariana (\%) & 65,3 & 54,2 & $<0,05$ \\
\hline IG média (SG) & 33,4 & 34,2 & $<0,05$ \\
\hline PN médio $(g)$ & 1943 & 2147 & $<0,001$ \\
\hline Índice de Apgar médio ao $5^{\circ}$ minuto & 9,4 & 9,5 & $>0,05$ \\
\hline Internamento em UCIN (\%) & 54,9 & 46,7 & $<0,05$ \\
\hline
\end{tabular}

g: Gramas; SG: Semanas de gestação

Tabela 3 - Divisão dos recém-nascidos por IG e PN

\begin{tabular}{|c|c|c|c|}
\hline & $\begin{array}{c}M C \\
(n=273)\end{array}$ & $\begin{array}{c}\text { BC } \\
(n=778)\end{array}$ & $p$ \\
\hline IG 24 a 26 SG (\%) & 4,4 & 3,3 & $>0,05$ \\
\hline IG 27 a 30 SG (\%) & 9,9 & 6,3 & $>0,05$ \\
\hline IG 31 a 33 SG (\%) & 20,1 & 17,9 & $>0,05$ \\
\hline IG 34 a 36 SG (\%) & 53,8 & 49,2 & $>0,05$ \\
\hline IG $\geq 37$ SG (\%) & 11,7 & 23,3 & $<0,001$ \\
\hline $\mathrm{PN}<1000 \mathrm{~g}(\%)$ & 10,6 & 4,8 & $<0,001$ \\
\hline PN 1000 a 1499 g (\%) & 11,0 & 9,5 & $>0,05$ \\
\hline PN 1500 a 2499 g (\%) & 62,6 & 57,7 & $>0,05$ \\
\hline$P N \geq 2500 \mathrm{~g}(\%)$ & 15,8 & 28,0 & $<0,001$ \\
\hline
\end{tabular}

g: Gramas; SG: Semanas de gestação 
A maior taxa de internamento ocorreu no grupo monocoriónico $(54,9$ vs $46,7 \%, p<0,05)$. A duração média de internamento foi semelhante (MC: 19,2 dias; BC: 16,2 dias; $p>$ $0,05)$.

Os gémeos monocoriónicos tiveram uma incidência mais elevada de doença de membrana hialina $(\mathrm{DMH})(7,0$ vs $4,0 \%, p<0,05)$, sépsis $(10,3$ vs $5,8 \%, p<0,05)$ e anemia $(9,5$ vs $5,4 \%, p<0,05)$. Não se encontrou diferença estatisticamente significativa relativamente à ocorrência de enterocolite necrotizante, HPIV, ROP ou hiperbilirrubinémia (Tabela 4).

Houve uma maior incidência de óbitos fetais na gestação $M C(2,8$ vs $1,5 \%, p<0,05)$, dos quais $50 \%$ (4/8) eram casos de STFF. Registou-se um caso de hidrópsia fetal (gestação $\mathrm{BC}$ ), tendo sido as restantes mortes de causa indeterminada. Também na primeira semana de vida se verificou uma maior percentagem de mortes no grupo monocoriónico $(2,4$ vs $1,4 \%, p<0,05)$, totalizando uma mortalidade perinatal de 5,2\% por oposição a 2,9\% nos RN do grupo bicoriónico $(p<0,05)$ (Tabela 5).

\section{DISCUSSÃO}

Ao longo dos últimos anos tem-se verificado um aumento progressivo do número (absoluto) de gémeos bem como da taxa de gestação múltipla, à semelhança do que se tem constatado no resto do mundo. ${ }^{2,3,6} \mathrm{O}$ recurso a técnicas de PMA muito tem contribuído para esta realidade. .,9,13 $^{6}$ No nosso estudo, não só a PMA mas também o avançar da idade materna se revelaram fatores determinantes da corionicidade pois mesmo analisando apenas as gestações espontâneas se manteve uma diferença evidente na idade materna média entre os grupos, mais elevada na gravidez BC. ${ }^{3,13}$

A incidência de RCIU foi superior na gestação $M C$, como esperado. ${ }^{1,11,17}$ A STFF foi documentada em $11,1 \%$ das gestações $\mathrm{MC}$, o que está de acordo com o reportado por outros centros. ${ }^{10-12,16,17,22}$ À semelhança do que foi descrito por outro estudo português ${ }^{1}$, encontrou-se uma maior incidência de cesariana na gravidez MC.

Também na gestação MC se verificou uma maior incidência de PPT e um menor PN médio, o que está de acordo com a literatura. ${ }^{1,10,11,13,16,23}$ Estes parâmetros influenciaram, com elevada probabilidade, a maior taxa de admissão em UCIN destes RN. ${ }^{13,17}$

A corionicidade parece ter também um papel determinante na morbilidade neonatal: na nossa amostra, os gémeos monocoriónicos apresentaram maior incidência de $\mathrm{DMH},{ }^{1,3,17}$ sépsis $^{3}$ e anemia. Também a HPIV foi mais prevalente neste grupo, ${ }^{1,10,11,17}$ embora a diferença não tenha sido estatisticamente significativa.

Vários estudos têm evidenciado um maior risco de morte fetal e perinatal na gestação MC comparativamente com a $B C$ e a unifetal, ${ }^{1,4,10-13,16,23}$ em relação com maior incidência de RCIU, CFD e STFF no primeiro grupo..$^{4,16,17,22}$ No entanto, algumas séries mostram que mesmo as gestações MC não complicadas apresentam um risco de morte consideravelmente superior, ${ }^{24,25}$ o que leva a crer que a história natural da gestação múltipla e, em particular, da MC não seja ainda completamente conhecida. , $22^{2}$

Este estudo destaca o impacto que a gemelaridade tem numa maternidade central a vários níveis: no número total de nascimentos, no âmbito dos RN muito baixo peso, na prematuridade e nas admissões em UCIN, o que vai ao encontro do que é descrito por outros autores. . $^{2,3,11,12,22,26}$

Como limitações deste trabalho salientam-se a natureza retrospetiva da análise e a dimensão da amostra que, embora grande para o universo nacional, não permite números expressivos que possibilitem determinadas comparações entre os grupos, nomeadamente a incidência de morbilidade neurológica em função da corionicidade. 1,10,11,17 $^{-17}$

Tabela 4 - Morbilidade neonatal

\begin{tabular}{|c|c|c|c|}
\hline & $\begin{array}{c}M C \\
(n=273)\end{array}$ & $\begin{array}{c}\text { BC } \\
(n=778)\end{array}$ & $p$ \\
\hline DMH (\%) & 7,0 & 4,0 & $<0,05$ \\
\hline Sépsis (\%) & 10,3 & 5,8 & $<0,05$ \\
\hline Anemia (\%) & 9,5 & 5,4 & $<0,05$ \\
\hline Enterocolite necrotizante (\%) & 2,6 & 0,9 & $>0,05$ \\
\hline HPIV (\%) & 3,3 & 1,8 & $>0,05$ \\
\hline ROP (\%) & 2,2 & 0,9 & $>0,05$ \\
\hline Hiperbilirrubinémia (\%) & 40,7 & 35,9 & $>0,05$ \\
\hline
\end{tabular}

Tabela 5 - Mortalidade perinatal

\begin{tabular}{|c|c|c|c|}
\hline & $\begin{array}{c}M C \\
(n=286)\end{array}$ & $\begin{array}{c}B C \\
(n=789)\end{array}$ & $p$ \\
\hline Óbitos fetais de 28 ou mais SG (\%) & 2,8 & 1,5 & $<0,05$ \\
\hline Mortalidade neonatal precoce (1 - 7 dias de vida) (\%) & 2,4 & 1,4 & $<0,05$ \\
\hline Mortalidade neonatal tardia (8 - 28 dias de vida) (\%) & 1,0 & 0,8 & $>0,05$ \\
\hline Mortalidade perinatal & 5,2 & 2,9 & $<0,05$ \\
\hline
\end{tabular}

SG: Semanas de gestação 
Nos gémeos fruto de gestação $\mathrm{MC}$, sobretudo nos que foram sujeitos a STFF, está descrita uma maior incidência de morbilidade neurológica (paralisia cerebral, perturbações do espetro do autismo, perturbação de hiperatividade e défice de atenção), ${ }^{13,27,28}$ pelo que seriam úteis estudos prospetivos que permitissem avaliar o desenvolvimento psico-motor destas crianças em idade escolar.

\section{CONCLUSÕES}

Em conclusão, a gemelaridade tem atualmente um importante impacto nos nascimentos, pelo que seria interessante desenvolver protocolos que uniformizassem a prática clínica na abordagem a estes RN. Os gémeos monocoriónicos, em particular, constituem um desafio quer para obstetras quer para neonatologistas, devendo a vigilância pré-natal e o parto ser realizados em centros de referência.

\section{AGRADECIMENTOS}

Os autores gostariam de agradecer ao Serviço de Obstetrícia da Maternidade Daniel de Matos do Centro Hospitalar e Universitário de Coimbra pelo contributo na vigilância pré-natal.

\section{REFERÊNCIAS}

1. Manso P, Vaz A, Taborda A, Silva IS. Chorionicity and perinatal complications in twin pregnancy: a 10 years case series. Acta Med Port. 2011;24:695-8.

2. Hayes EJ, Paul D, Ness A, Mackley A, Berghella V. Very-low-birthweight neonates: do outcomes differ in multiple compared with singleton gestations? Am J Perinatol. 2007;24:373-6.

3. Burgess JL, Unal ER, Nietert PJ, Newman RB. Risk of late-preterm stillbirth and neonatal morbidity for monochorionic and dichorionic twins. Am J Obstet Gynecol. 2014;210:578.e1-9.

4. Russo FM, Pozzi E, Pelizzoni F, Todyrenchuk L, Bernasconi DP Cozzolino S, et al. Stillbirths in singletons, dichorionic and monochorionic twins: a comparison of risks and causes. Eur J Obstet Gynecol Reprod Biol. 2013;170:131-6.

5. Endres L, Wilkins I. Epidemiology and biology of multiple gestations. Clin Perinatol. 2005;32:301-14.

6. Rodrigues CT, Branco MR, Ferreira ID, Nordeste A, Fonseca M, Taborda A, et al. Multiple gestation epidemiology -15 years survey. Acta Med Port. 2005;18:107-11.

7. Almeida P, Domingues AP, Belo A, Fonseca E, Moura P. Triplet pregnancies: perinatal outcome evolution. Rev Bras Ginecol Obstet. 2014;36:393-7.

8. Domingues AP, Fonseca E, Belo A, Moura P. Twins prematurity - the influence of prenatal surveillance. J Matern Fetal Neonatal Med. 2015;28:1108-11.

9. Adegbite AL, Ward SB, Bajoria R. Perinatal outcome of spontaneously conceived triplet pregnancies in relation to chorionicity. Am J Obstet Gynecol. 2005;193:1463-71.

10. Trevett T, Johnson A. Monochorionic twin pregnancies. Clin Perinatol. 2005;32:475-94.

11. Rao A, Sairam S, Shehata H. Obstetric complications of twin pregnancies. Best Pract Res Clin Obstet Gynaecol. 2004;18:557-76.

12. Gomella TL, Cunningham MD. Neonatology: management, procedures, on call problems, diseases, and drugs. $6^{\text {th }}$ ed. New York: McGraw-Hill Companies, Inc; 2009.

13. Shrim A, Weisz B, Gindes L, Gagnon R. Parameters associated with outcome in third trimester monochorionic diamniotic twin pregnancies. J Obstet Gynaecol Can. 2010;32:429-34.

14. Ville Y. Chorionicity and mode of fertilization: a long-standing misconception. Ultrasound Obstet Gynecol. 2006;28:635-6.

15. Parazzini F, Cipriani S, Bianchi S, Bulfoni C, Bortolus R, Somigliana E. Risk of monozygotic twins after assisted reproduction: a populationbased approach. Twin Res Hum Genet. 2016;19:72-6.

\section{OBSERVAÇÕES}

Parte do trabalho foi apresentado no $1^{\text {st }}$ Congress of Joint European Neonatal Societies (jENS) em Budapeste 16 a 20 de setembro de 2015.

\section{PROTECÇÃO DE PESSOAS E ANIMAIS}

Os autores declaram que os procedimentos seguidos estavam de acordo com os regulamentos estabelecidos pelos responsáveis da Comissão de Investigação Clínica e Ética e de acordo com a Declaração de Helsínquia da Associação Médica Mundial.

\section{CONFIDENCIALIDADE DOS DADOS}

Os autores declaram ter seguido os protocolos do seu centro de trabalho acerca da publicação de dados.

\section{CONFLITOS DE INTERESSE}

Os autores declaram não terem qualquer conflito de interesse relativamente ao presente artigo.

\section{FONTES DE FINANCIAMENTO}

Não existiram fontes externas de financiamento para a realização deste artigo.

16. Lewi L, Jani J, Blickstein I, Huber A, Gucciardo L, Van Mieghem T, et al The outcome of monochorionic diamniotic twin gestations in the era of invasive fetal therapy: a prospective cohort study. Am J Obstet Gynecol. 2008;199:514.e1-8.

17. Acosta-Rojas R, Becker J, Munoz-Abellana B, Ruiz C, Carreras E, Gratacos $E$, et al. Twin chorionicity and the risk of adverse perinatal outcome. Int J Gynaecol Obstet. 2007;96:98-102.

18. Gratacós E, Deprest J. Current experience with fetoscopy and the Eurofoetus registry for fetoscopic procedures. Eur J Obstet Gynecol Reprod Biol. 2000;92:151-9.

19. Volpe JJ. Neurology of the newborn. $5^{\text {th }}$ ed. Philadelphia: WB Saunders Elsevier; 2008.

20. Secção de Neonatologia da Sociedade Portuguesa de Pediatria, 2013. Consenso clínico: anemia da prematuridade. [consultado 2015 set 10]. Disponível em:http://www.lusoneonatologia.com/pt/consenso/73/ consensos-e-recomendacoes/.

21. International Committee for the Classification of Retinopathy of Prematurity. The international classification of retinopathy of prematurity revisited. Arch Ophthalmol. 2005;123:991-9.

22. Smith NA, Wilkins-Haug L, Santolaya-Forgas J, Acker D, Economy $\mathrm{KE}$, Benson $\mathrm{CB}$, et al. Contemporary management of monochorionic diamniotic twins: outcomes and delivery recommendations revisited. Am J Obstet Gynecol. 2010;203:133.e1-6.

23. Carroll SG, Tyfield L, Reeve L, Porter H, Soothill P, Kyle PM. Is zygosity or chorionicity the main determinant of fetal outcome in twin pregnancies? Am J Obstet Gynecol. 2005;193:757-61.

24. Barigye O, Pasquini L, Galea P, Chambers H, Chappell L, Fisk NM. High risk of unexpected late fetal death in monochorionic twins despite intensive ultrasound surveillance: a cohort study. PLoS Med. 2005;2:e172.

25. McPherson JA, Odibo AO, Shanks AL, Roehl KA, Macones GA, Cahill AG. Impact of chorionicity on risk and timing of intrauterine fetal demise in twin pregnancies. Am J Obstet Gynecol. 2012;207:190.e1-6.

26. Martin JA, Hamilton BE, Sutton PD, Ventura SJ, Menacker F, Kirmeyer S, et al. Births: final data for 2005. Natl Vital Stat Rep. 2007;56:1-103.

27. Mclntosh J, Meriki N, Joshi A, Biggs V, Welsh AW, Challis D, et al. Long term developmental outcomes of pre-school age children following laser surgery for twin-to-twin transfusion syndrome. Early Hum Dev. 2014;90:837-42.

28. Rand L, Eddleman KA, Stone J. Long-term outcomes in multiple gestations. Clin Perinatol. 2005;32:495-513. 